\title{
UNA UNIVERSIDAD SOLO PARA MUJERES EN EL SIGLO XXI
}

\author{
A XXI CENTURY UNIVERSITY ONLY FOR WOMEN
}

Entrevista a la Dra. Victoria García García, Vicerrectora Académica - UNIFÉ ${ }^{1}$

Por: Dra. Olga González Sarmiento ${ }^{2}$

\section{RESUMEN}

En esta entrevista la Dra. Victoria García García, habla de la trayectoria de la UNIFÉ, como Universidad de la Mujer en el Perú, a lo largo de sus 50 años de vida institucional.

La Dra. García, es la actual Vicerrectora Académica de la UNIFÉ; y, estudió Psicología en las primeras promociones de la Universidad. Tiene una trayectoria de 37 años como docente universitaria. Ha recibido el Premio a la Docencia Universitaria y el Premio Nacional de Psicología, en los años 2005 y 2009 respectivamente, ambos premios otorgados por el Colegio de Psicólogos del Perú.

Para hacer más claro el tema tratado, la entrevista ha sido planteada en 12 interrogantes.

\section{ABSTRACT}

In the present interview, Ph.D. Victoria García García talks about the evolution of UNIFE University, as University of Women in Peru, throughout 50 years of institutional life.

Currently, Ph.D. García is the Academic Deputy Vice - Chancellor of UNIFE University. She studied Psychology in one of the first proms of the university. She has 37 years of trajectory as university teacher. In 2005 she receives the Distinguished Teaching University Award and in 2009 she received the National Psychology Award, both prizes awarded by the Peruvian Collegue of Psychologist.

To make clear the treated subject, the interview was raised in 12 questions.

\section{1. ¿Por qué la UNIFÉ es una universidad exclusivamente para mujeres en pleno siglo XXI?}

Agradezco tu pregunta, yo podría responderte: ¿'y por qué no? Para mí es una alternativa de educación diferenciada totalmente válida. Y, la mejor respuesta es que UNIFÉ cumple 50 años de existencia académica ininterrumpida. Una universidad con prestigio ganado gracias a su compromiso con la realidad nacional y con la formación de las estudiantes, dado que apuesta en forma decidida por la calidad educativa.

Como toda universidad, la UNIFÉ está pendiente del pulso social y debe responder a la problemática o inquietudes de su entorno, y sin lugar a dudas, es evidente la necesidad de impulsar la educación de la mujer. Punto en la agenda mundial de la ONU y de la OEA, entre otros organismos internacionales.

1 Doctora en Psicología, Docente Principal del Departamento de Psicología de la UNIFÉ, Vicerrectora Académica de la UNIFÉ, Past Decana de la Facultad de Psicología de la UNIFÉ, ha publicado numerosos artículos, ha presentado ponencias en el Perú y en el extranjero.

2 Doctora en Educación y Magíster en Tecnología Educativa, con segunda especialidad en Formación Magisterial. Coordinadora de la Unidad de Investigación de la Facultad de Educación de la Universidad Femenina del Sagrado Corazón, Docente universitaria y Consultora educativa. 
Dentro de nuestro país, somos una sociedad en la cual el tema de la equidad de la mujer es todavía sensible, sobre todo en el entorno de la educación.

Si bien es cierto UNIFÉ se crea en 1962 como una entidad académica para impulsar la formación de la mujer de ese entonces, actualmente el panorama ha cambiado. La demanda de las mujeres por la formación universitaria se ha incrementado. Se evidencia en diversas fuentes, por ejemplo, Claudio Rama (2009) en su libro sobre la universidad se refiere a ese punto; además, las estadísticas del INEI lo expresan. En el último censo universitario del 2010 en el país se aprecia que la población femenina universitaria tiene un porcentaje de $49 \%$ y la masculina de un $51 \%$. Esas cifras permiten concluir que la participación de la mujer en la educación en estos años definitivamente significa un cambio en su perfil en los últimos tiempos.

¿Por qué entonces una universidad para mujeres en el siglo XXI, 50 años después? Porque creo que el desarrollo o éxito de la mujer no está solo en el acceso a la productividad, sino en cómo las propias mujeres queremos ser transformadas, es decir, en la propia construcción de nuestro esquema mental como mujeres respecto al progreso, a la autonomía y al desarrollo. Entonces, yo puedo decir que el siglo XXI es un siglo en el cual la mujer conquista su promoción y desarrollo intelectual y laboral, pero paralelamente debe velar por su desarrollo personal y emocional.

Por ende, el éxito de un posicionamiento ocupacional en un trabajo, de su acceso a la vida universitaria, de su profesionalización no lo es todo. Creo que en el caso de la mujer, un punto importante es el esquema que ella misma tenga de su rol como mujer. Por eso, yo formularía una pregunta... ¿Ese cambio modifica el esquema conceptual de ser mujer? Creo que allí radica una respuesta fundamental, y creo que no se ha dado, por lo menos en nuestro país. Si bien es cierto que hay avances en una mayor participación resultado de su profesionalización, sin embargo, el esquema mental de la mujer no ha cambiado, la mujer sigue siendo concebida y sintiéndose "todista".
Asume las responsabilidades de la casa, de la carrera, de la profesión, del trabajo, de la sociedad y aún no está preparada todavía para encajarlas necesariamente. Las propias mujeres no pueden manejar su propio rol, evidentemente es problemático. Entonces en este sentido, sí es importante que exista una universidad femenina, una universidad para la mujer con un espacio académico para ella, un espacio que le permita fundamentalmente reflexionar en sí misma.

Uno no viene a la universidad necesariamente para buscar una meta de desarrollo personal afectivo emocional, si la encuentras es parte de una dinámica, pero no es el objetivo de la universidad ni la razón de esta, entonces partiendo de esto, ¿por qué no podría haber una universidad diferenciada solo de hombres o solo de mujeres? En ese sentido, UNIFÉ es un espacio para la formación académica de la mujer para ayudarla a reflexionar sobre sí misma, sobre sus diferentes roles en la sociedad y darle una formación integral que pueda serle útil a la sociedad.

Quiero agregar además que nosotros en la universidad no tenemos una opción feminista, y subrayo esto, somos conscientes que la sociedad es mixta, que hombre y mujer son complementarios, no son sexos opuestos, y esa es la perspectiva que enfocamos en nuestro modelo educativo. Entonces, volvemos a la pregunta inicial, ¿debe haber una universidad exclusivamente para la mujer en pleno siglo XXI? ¿Por qué no? Eso va a depender de las sociedades, de la evolución y el desarrollo y de la propia aceptación de la mujer de su rol como tal, y yo creo que en nuestro país todavía hay mucho que mejorar.

\section{Diría usted que en la formación que ofrece la UNIFÉ también se está propiciando ese cambio de mentalidad en las estudiantes?}

Yo diría que no es que promueva directamente o propicie que ese cambio se dé a través de asignaturas o de actividades específicas, yo creo que el espacio de convivir en grupo de mujeres, personas del mismo género, es un gran laboratorio, ese es el mejor entrenamiento. En 
Estados Unidos es muy característico eso de los Training Groups (grupos de entrenamiento). Acá no tenemos esa experiencia, este es el laboratorio, las estudiantes pasan 5 o 6 años (dependiendo de la carrera) en grupos de mujeres de todo tipo, ese es el mejor entrenamiento para definir roles, es el mejor laboratorio para esa situación. Entonces creo que allí está, eso las va a enriquecer, tenemos muchas mujeres exalumnas de la universidad todas realizadas personal y profesionalmente, no es una universidad donde la mujer entre aquí y olvide la parte de desarrollo personal. Yo me preguntaría, si se ha hecho alguna investigación, o tal vez es eso lo que nos falta: ¿Cuántas personas egresadas de universidades mixtas no han logrado un desarrollo personal en su vida? y ¿̇cuántas profesionales egresadas de nuestra universidad han logrado el desarrollo personal y profesional?

\section{Respecto a su respuesta a la primera pregunta, ¿qué diferencias posee, entonces, la educación destinada al género femenino con respecto a la que se ofrece en universidades donde se forman a ambos sexo?}

En realidad yo preferiría no hacer un juicio de valor, o sea no decir si es mejor o no lo es, pero sí te puedo responder que se han hecho estudios en Estados Unidos, donde tienen más de 82 instituciones femeninas de nivel superior y se ha descubierto que estudiar en una universidad de mujeres influye positivamente en su formación; esto es una publicación que hace la Red de Universidades Femeninas de Estados Unidos. Quiero decir que esa Red Mundial de Universidades Femeninas ya nos ha invitado a ser parte de ella y una de sus principales líderes es Hillary Clinton, ella es una egresada de una universidad femenina. Corea tiene ocho universidades de la mujer, también las tienen países como India, Japón. Es un tema que tiene que ver con la sociedad, la evolución, la cultura, este un punto importante a tenerse en cuenta. Entonces si tú me preguntas, ¿por qué puede ser mejor? Yo te contesto, este estudio hecho en el 2009 por esta Red de Universidades Femeninas de Estados Unidos, lo comprueba, lo comprueban ellos, lo comprobamos acá, mejora la autoestima, estimula la posibilidad de participación en clase al no estar el varón...

Al estar el varón surge la competencia, pero una competencia de género, aquí la chica no tiene que competir con el varón, no tiene que cuidarse que si le gusta, que si es agradable, porque la edad a la que ingresan a la universidad es cada vez menor y están en pleno proceso evolutivo (16-17 años), en el caso de universidades de mujeres al no estar el varón no les importa competir entre ellas. Tienen más oportunidades de desarrollar el liderazgo, te permite conocer mejor tu género, que era lo que te decía en la pregunta anterior, lo que beneficia a su autoconocimiento y es clave para el futuro.

Una clave para interactuar con los otros es conocerte a ti mismo, y no sé si en esto estoy hablando un poco por mi carrera, por mi propia formación como psicóloga, pero realmente lo creo, y en comparación con las universidades mixtas. Se ha visto, por ejemplo, que las mujeres se gradúan más rápidamente y suelen tener mayor satisfacción en relación a la experiencia universitaria que las que estudiaron en universidades mixtas. O sea, se ha hecho un estudio comparando a las que han estudiado en universidades femeninas y las que han terminado en universidades mixtas, resulta, quizá porque no se han complicado con parejas emocionales que tienen una mayor satisfacción al concluir sus estudios que las que han estado en universidades mixtas.

Y existe también un estudio de una española, Isabel Cuadrado en el 2004 que dice que el estar solamente en universidades femeninas ayuda a valorar la relación, la colaboración y la discusión, es decir les gusta el debate, todas estas competencias necesarias en el campo ocupacional. Entonces, ya hay estudios que están comprobando su efectividad.

\section{La mayoría dice: ¿por qué?, si es una sociedad de tipo mixto..., ¿́por qué razón?}

Claro, se quedan en la primera opción quizá y no profundizan. 
La Dra. Yarviga Sashbrech, past Presidenta de la Asociación de Universidades Femeninas en Estados Unidos, presenta los estudios de todos los beneficios. Incluso hay una publicación de la universidad de Navarra con el título "Una universidad solo para mujeres..." (lee muy rápido un texto sobre el inicio de Harvard y finalmente termina hablando)... la Dra. Cuadrado dice "no creo que el modelo de universidades de mujeres deba ser reproducido en España pero tal vez nuestra política educativa deba apoyar proyectos escolares de educación diferenciada junto con los proyectos de educación actual, esto quiere decir que en la propia universidad de Navarra (España) ya lo están planteando.

Quiero decirte que en Estados Unidos, por otras razones, están volviendo en este momento a afianzar más la educación diferenciada por un tema de problemas, de prevenir un poco el embarazo pre adolescente, pero esos son otros temas, por ejemplo están trabajando como una alternativa desde el punto de vista de la educación en la sociedad norteamericana.

\section{UNIFÉ cumple en el presente año medio siglo de trabajo constante a favor de la educación de nuestro país, ¿cuáles son a su parecer aquellos aspectos donde se han realizado sus mejores y mayores aportes en el campo educativo, y cuáles son aquellos que aún quedan por mejorar?}

Desde mi punto de vista, el mejor aporte son nuestras egresadas, nosotros tenemos actualmente cerca de 12200 tituladas que están bien ubicadas en el mercado laboral y gracias a ellas hemos logrado nuestro posicionamiento académico, que es importante, para mí ese es el principal aporte a la sociedad, yo diría una formación integral que busca el desarrollo profesional y personal.

Nuestras carreras de nivel de pregrado se desarrollan en una estructura curricular integral con cursos de estudios generales, de axiología, aparte de los cursos de especialidad. También tenemos cursos de desarrollo social, como universidad católica somos una universidad que busca sensibilizar al estudiante para apoyar a los menos favorecidos. Puede ser que el estudiante cuando está en aula, quizá no sea consciente directamente de estas ventajas; la estudiante entra a la universidad, se sienta y sigue un plan curricular. Quizá ella no está haciendo el análisis que hemos hecho nosotros para obtener una estructura curricular que la tenemos y sostenemos durante 50 años, obviamente se han ido actualizando los cursos básicos, pero está la estructura.

Yo me acuerdo, por ejemplo, en los primeros años de universidad siempre hubo estudios generales y había pocas universidades que los tenían, ahora, en el país solo nos quedamos dos universidades privadas con dos años de estudios generales con una diferencia sustancial. En la otra universidad, después de dos años pasan a la especialidad, nosotros desde primer ciclo lo que hacemos es integrarlo a la especialidad, por eso lo llamamos estudios generales y pre especialidad. ¿Cuál es la tendencia actual de las universidades? miran a la necesidad de dar una cultura general en la formación profesional, nosotros no cambiamos eso, no quitamos los cursos de filosofía, de teología, puede ser que la estudiante en algún momento diga ¿̇y esto para qué? Pero, cuando salen y egresan lo reconocen, porque gracias al estudio y profundización de muchos temas de la humanidad pueden entender mejor la realidad. Entonces, yo diría la estructura que tenemos nosotros es un aporte a la educación, es un aporte a la formación porque hemos logrado integrar desarrollo profesional, científico, cultural, actividades, axiología, queda claro que los valores no se aprenden en un curso, se aprenden a lo largo de la vida, pero les damos la facilidad o la posibilidad de esto. ¿Cómo?, dentro de las actividades de desarrollo social.

Otra gran fortaleza de UNIFÉ en estos 50 años ha sido su proyección social. Esto es fundamental porque la proyección social se cumple en UNIFÉ como un área académica fundamental, tú sabes que son cuatro básicamente las áreas fundamentales del área académica: la formación profesional, la investigación, la proyección social y la extensión universitaria. Bueno, la UNIFÉ puede decir que cumple a cabalidad la 
proyección social porque es un eje transversal a lo largo de todo el plan curricular. La estudiante, desde el primer ciclo hasta que termina trabaja con el tema de proyección social, entonces yo creo que ese es un punto importante, y va de la mano con nuestra misión. Actualmente, estamos en Jicamarca, en Villa el Salvador, hemos estado 15 años en Chilca y así estamos apoyando zonas de nuestra sociedad donde pensamos que nuestras estudiantes pueden volcar sus servicios, es parte de nuestra vocación.

Evidentemente, siempre hay aspectos que mejorar, ese es el otro lado. Yo diría que toda organización evoluciona, lo que quiero decir es que siempre tenemos que mejorar, debemos pensar que todas las organizaciones van cambiando, logran determinadas metas y tienen que ir por otras. Creo que eso es parte de la dinámica y nosotros tenemos facetas que mejorar, por ejemplo el de promover la investigación, y no solo las nuevas sino también publicitar las que tenemos, creo que es un tema pendiente en nuestra agenda y hay que reconocerlo.

Otro tema es ampliar la oferta educativa con carreras más actuales, más del momento, creo que quizá hemos optado por carreras tradicionales, clásicas porque son carreras que en el fondo son más elegidas por el género femenino: educación, psicología, nutrición, traducción, donde generalmente el mayor número de profesionales son mujeres, pero hoy tenemos ingeniería, arquitectura, administración de negocios y creo que debemos seguir optando por otras carreras más novedosas, más vigentes, creo que allí es otra agenda la que tenemos que desarrollar.

Otro punto es lograr gestionar y sistematizar el monitoreo permanente, yo en esto no sé si voy a discrepar o no con las tendencias actuales, creo que es necesaria la acreditación, pero hay acreditación y acreditación y hay autoevaluación y autoevaluación, yo no creo que las universidades debamos acreditarnos y autoevaluarnos para decir que estamos acreditadas, obtener los diplomas, publicarlos en los periódicos, sacarnos una foto, pagar a las agencias para acreditarnos, porque creo que la acreditación no debe ser eso, creo que las propias universidades debemos desarrollar un aporte de una cultura de autoevaluación permanente.

La autorregulación no debe hacerse para estar en un ranking, para pagar a una aseguradora o a un equipo o para decir 'mira ya acredité a todas mis carreras', porque eso es en un papel, yo creo que la mejor acreditación está en la formación que tú puedes ver en nuestras profesionales, en la calidad... Sin embargo, creo que es necesario en un momento en el que hay tantas universidades, como tú misma lo has mencionado en tu pregunta, donde la competencia es muy grande, entonces es conveniente decir iMira aquí estamos!, ¿quieres ver mi calidad? Allí están mis egresadas y todo el impacto social que generan! Pero sí creo que debemos gestionar mejor ese tema, porque dadas las competencias, no digo que no se deban sacar estos diplomas pero creo que sí es necesario acceder a determinados estándares y otra tarea pendiente es realizar un trabajo conjunto con la asociación de egresadas

Para resumirte creo que lo mejor que tiene UNIFÉ es su formación profesional y su proyección social, creo que lo que tenemos que mejorar es la investigación, es darnos a conocer a los demás, creo que allí hay una gran desventaja, un déficit de nuestra parte; y otro gran tema es mirar alrededor y gestionar mejor esta cultura de monitoreo permanente.

6. He tenido la oportunidad de conversar en otros medios universitarios y he contado experiencias que se realizan acá en la UNIFÉ y me dicen ¿Por qué no lo publican? Con la mitad de todo eso, tal universidad lo hubiera puesto en los medios, hacemos muchas cosas, pero nadie lo sabe, entonces una persona me dijo "si nadie lo sabe es como si no se hiciera", y no es que no se haga, sino que no se publica. Cuando Ud. mencionaba que había identificado esta carencia o esa tarea por hacer, pensaba que es un punto de partida para lo ya mucho que se está haciendo y los cambios que se están realizando. 
Yo te contaré que en una oportunidad una docente había publicado un libro y viene y me dice "pero el libro no se difunde, no lo sacan a venta, no se promueve" y le respondo, ¿quién va a promover tu libro? Claro, la universidad debería promoverlo, pero si yo estoy en una universidad y yo he publicado un libro, ¿quién es la primera interesada en difundirlo? iEl autor! Hoy por hoy hay los medios y los canales y creo que la posibilidad de hacerlo es más fácil que antes y creo que en esa línea tenemos que hacerlo.

\section{Hoy en día se habla muchísimo sobre el desarrollo de las capacidades en la persona, gracias a la intervención de la educación, según su opinión ¿cuáles son las capacidades más importantes y urgentes por las que se debería trabajar con mayor ahínco desde la Universidad?}

Voy enfocar esta respuesta no desde UNIFÉ, sino desde la Universidad en general, desde la universidad peruana. Yo creo que... bueno lo que digo tal vez no es novedoso, lo vienen diciendo muchos expertos nacionales $e$ internacionales, pero creo en ello y por eso lo hago mío en este momento, creo que se deben propiciar las metodologías activas y colaborativas en el proceso de enseñanza y aprendizaje porque sólo esto va a propiciar personas participativas, dinámicas, colaborativas, creativas, capaces de resolver problemas, de poder hacer los cambios en la sociedad. Hoy la educación superior debe facilitar estudiantes proactivos, innovadores, con valores, porque son los que van a hacer el cambio, son los que definitivamente van a transformar la sociedad o van a tener el soporte fundamental o el peso de una sociedad que evoluciona con una gran rapidez.

Sin embargo las universidades peruanas, como una crítica en general, tienen mucho alumnado $y$ es un poco difícil dar ese trato personal necesario, en mi opinión, que requiere el estudiante para desarrollar esas competencias y se necesitan para lograr una pedagogía colaborativa, participativa, de ciudadanos responsables y competentes. Sin embargo, yo te diría que en UNIFÉ sí es posible, porque no somos una universidad masiva, no somos una universidad de mucha gente, ofrecemos una mejor posibilidad en aula para ese diálogo y esa relación docente-estudiante, una relación más interactiva.

Yo diría que el éxito para el futuro está en eso, no tanto en el conocimiento del profesor sino en la actitud del profesor, que contagien actitudes y permitan una interacción de actitudes que creo que es un tema fundamental. Tenemos que fortificar a las personas, la sociedad cambia rápidamente, la persona debe estar clara pero debe ser dinámica, debe sentirse bien, debe sentirse con bienestar para poder trabajar por nuestra sociedad.

8. Existen un poco más de 100 universidades que ofrecen un sinnúmero de carreras en nuestro país, sin embargo, se cuestionan muchas veces los niveles de calidad en el servicio educativo que se ofrece en alguna de ellas. Según su experiencia y conocimiento, ¿cuáles son los estándares de calidad más importantes que debe ofrecer una universidad comprometida con educación?

Para mí el tema del estándar es un patrón, es un poco encorsetar a la institución para el logro de algo, sin embargo, y como te decía creo que debe haber un monitoreo permanente autorregulado general de la propia institución, en este sentido, yo insisto, para mí lo más importante es la formación profesionaly todo lo que signifique este estándar o esta área fundamental de la formación profesional a través de las metodologías de la enseñanza o del aprendizaje, a través del rol del docente, que no sea solamente un conocedor de su profesión sino que sea un comunicador, una persona que promueva la investigación, pero en todos los campos, científicos y tecnológicos, y que esa investigación se dedique fundamentalmente a trabajar o a explorar; a explorar los campos productivos de nuestro país: la minería, la agricultura, el turismo.

Hay países donde no se dan todas las carreras, se dan las carreras que el país requiere, aquí tenemos una oferta muy diversa, pero creo que estamos desperdiciando formación 
de profesionales en lo que es nuestro: la agricultura, la minería, el turismo y tantos otros oficios, aquí todo el mundo va a las universidades y en nuestro perfil como país somos muy hábiles con las manos, la parte artesanal y eso lo hemos demostrado, somos grandes gestores de empresa, somos muy productivos a ese nivel, entonces yo creo que allí es donde se debe ir con la investigación; con esto no quiero decir que no entremos al campo de la genética, ni a aspectos más avanzados como la nanotecnología, pero creo que entrar por lo que nuestro país requiere, yo diría que allí hay un tema dentro de la investigación.

9. Y no solamente en el tema de la investigación, sino ahora que usted hablaba acerca de las nuevas carreras de UNIFÉ, quien sabe, ¿tal vez este sea un punto de partida para la nueva oferta de carreras?

Justamente estamos en ese análisis para ver nuevas opciones en función de lo que el país necesita: turismo, agricultura, textilería, China no es el único país capaz de producir estas telas grabadas o pintadas, acá tenemos mucho potencial, hay que pensar en estos nuevos campos donde desarrollarse.

El otro tema es fomentar la internacionalización, pero yo diría no sólo la internacionalización, sino también la regionalización, antes de conocer otros países es importante conocer nuestra identidad cultural que es muy rica, nuestra diversidad, propiciar una movilidad interregional, porque una cosa es lo que he oído, visto o conversado; y otra cosa es la que te ayuda a conocer a nuestro país a nuestra realidad. Entonces, hablamos de una movilidad universitaria que no solo vea la internacionalización, sino también que vea la regionalización. Y lo que ya te decía sobre la cultura de la calidad permanente.

10. Una de las más interesantes propuestas con que cuenta un UNIFÉ hoy en día es el Programa PROMEL, dirigido a mujeres que se encuentran trabajando y que desean estudiar una carrera en un tiempo más breve que lo habitual, en este contexto ¿qué características debería tener la formación destinada a personas que presentan un perfil muy distinto al de las jóvenes que estudian su primera carrera?

Como bien lo has dicho el perfil de la estudiante es completamente distinto, $y$ en este sentido nosotros no podemos pretender que el plan de estudios, las metodologías de enseñanza y aprendizaje, el rol del maestro, las evaluaciones, etc. sean exactamente igual a las de las estudiantes regulares o mucho más jóvenes o sin experiencia de otras carreras o sin mayor "experiencia de vida" y lo pongo entre comillas porque la edad no es sinónimo de mayor o menor experiencia.

Sí, definitivamente este es un programa para mujeres con experiencia laboral que debe tener un diseño distinto, por ejemplo, yo creo que en primer lugar en cuanto al plan de estudios, el contenido ya programático, en cuanto al sílabo debe ser debidamente seleccionado, porque como es menos tiempo de estudios, no le podemos llevar a la estudiante a que tenga un gran cúmulo de información y que luego no pueda procesarla porque no le va a dar el tiempo, cosa que sí sucede con una alumna regular, por ejemplo con nuestras alumnas de día, casi todos nuestros cursos tienen teorías, seminario y práctica (cinco o cuatro horas), en el programa de la noche no se puede dar todo esto, entonces allí hay una "pérdida", pero en mi opinión puede ser recuperada, dependiendo de la metodología del profesor, si por ejemplo un profesor da conocimiento cuatro o cinco semanas, luego les da la opción del diálogo, de intercambiar ideas, les pide trabajos prácticos y sobre todo utiliza las vivencias de las propias estudiantes, porque ellas ya son personas que están trabajando en otros campos profesionales, por lo tanto poseen una mayor riqueza, pero para eso se requiere profesores debidamente preparados con buena metodología, con buena didáctica, si tú me preguntas, ¿̇cómo debe ser? podría ser el mismo plan de estudios en cuanto a cursos pero con una metodología distinta, con una forma de evaluar distintas, no se puede evaluar con pruebas memorísticas, porque por razones obvias son personas que tienen mucha información que retener por lo tanto 
es más importante ver su vivencia en relación al contenido que están trabajando, quizá un trabajo, una experiencia vivencial, una casuística, el análisis situacional, el famoso ABP (Aprendizaje Basado en Problemas) podría ser una alternativa. Y el otro tema es utilizar el debate y el diálogo permanente y utilizarlo como una forma de metodología, no para entrar en discusión sino como una forma de enriquecimiento.

\section{La Conferencia Mundial sobre Educación Superior realizada en París en el año 2009 abogaba porque las universidades en cada país se convirtieran en factores para el desarrollo, según su opinión, ¿qué cambios deberían darse en el quehacer universitario actual para que esta propuesta se convierta en una realidad palpable?}

Una vez más te hablo de la Universidad Peruana, no específicamente de UNIFÉ. La Universidad, primeramente, debe propiciar la investigación desde los primeros ciclos de estudios universitarios, yo no pienso que promover investigación en nuestro país implique únicamente, crear vicerrectorados de investigación, no creo que promover investigación en el país implique esperar a los últimos ciclos de la carrera..., para mí investigar es una estrategia de aprendizaje que debe utilizarse desde el colegio y debe seguir utilizándose a lo largo de toda la formación educativa, por tanto, si me llegan a un primer ciclo de cualquiera de las carreras debería estimularse la investigación formativa, desde los primeros ciclos, esto si la estudiante tiene esta posibilidad, esta alternativa en uno o dos o todos los cursos, investigar le va a resultar mucho más fácil, entonces, más adelante optar por una investigación aplicada de largo aliento que pueda servir para el país resultará más natural, más fácil.

Por otro lado, sería también interesante fomentar la titulación por tesis, no digo anular la forma que ya existe ahora sino llanamente fomentarla, hacerla más agradable; por alguna razón, a las estudiantes no les gusta preparar tesis, prefieren la vía más rápida y quizá sea porque no le encuentran un sentido, yo creo que esto es vital; lo otro, es lo que te decía hace un rato, incluir asignaturas de cultura general en los estudios básicos, que no la tienen todas las universidades, algunas han adoptado muchas asignaturas, pero no de cultura general, vemos que el nivel cultural de nuestros estudiantes en el país ha bajado drásticamente y no tengo que hablar de la expresión del lenguaje, la comprensión lectora, etc., que todo esto influye en la formación profesional.

El otro tema para mí importante es fomentar la responsabilidad universitaria, esto es vital en base a los voluntariados, en base a las actividades extracurriculares, la Universidad no es solamente seguir los cursos que llevo en una carrera, hay que saber vivir la vida universitaria.

La vida universitaria nos da conferencias, lo que llaman el aula abierta, es más fácil ver gente que hace fila para un concierto en cualquier otro lado y no hacen fila para escuchar a un premio Nobel o a un gran expositor, no lo consideran relevante, eso es un tema de responsabilidad, no es que el profesor nos lleve, es un tema de responsabilidad, hay que fomentarla, las universidades tenemos la obligación de fomentar esa responsabilidad universitaria a través de lo que te estoy diciendo, voluntariados, actividades extracurriculares, propiciarla los espacios y generar las carreras que están ligadas a nuestro país y principalmente, fomentar la creatividad y la innovación, creo que eso es un defecto de todo nuestro sistema educativo en general, no sólo de la Universidad.

El estudiante ha sido un gran dependiente y eso no lo podemos cambiar de la noche a la mañana, en cambio, si tú entras con una opción de un aprendizaje significativo, de un abp que dé una opción dinámica, van a decir y ¿ipara qué?

Creo que la Universidad debe ser un laboratorio de metodologías de aprendizaje, metodologías activas, metodologías tradicionales, diferentes formas, porque la sociedad no te va a dar una sola forma para responder, la sociedad tiene normas y patrones rígidos. 
Creo que un tema en la Universidad, aparte del conocimiento, es un tema formativo, pero un tema formativo en tu auto responsabilidad, es esa la educación a la que debemos mirar como un especie de autocontrol personal en una sociedad del siglo XXI, con una serie de estímulos, con una serie de situaciones, lo ideal sería tener personas que conozcan sus metas, sepan lo que quieren, a donde van, que es lo que desean, que haya una seguridad, una satisfacción y un bienestar y es eso lo que debemos propiciar, gente segura, que sepa qué es lo que vale, lo que siente realmente, que sea autor responsable y si se mete en un problema, asumirlo.

\section{Como profesional de la psicología que ha dedicado muchos años a la labor educativa, ¿podría decirme, según su perspectiva, ¿qué novedades puede to- davía ofrecer la educación a la persona y a la sociedad?}

Fundamentalmente para mí, en este siglo XXI, debe trabajarse mucho una pedagogía de la persona, donde la persona sea autónoma, sea más independiente, que los hombres y las mujeres puedan compartir y mejorar sus propias vidas, sus comunidades y su sociedad.
Educar ciudadanos éticos, responsables para evitar lo que muchas veces tenemos $y$ lo que vemos, gente que no tiene valores, profesionales susceptibles a la compra-venta de cargos, plagios, engaños, etc.

Para mí, la Universidad del siglo XXI debe preparar para la autoformación, la autoeducación y la autoevaluación, sólo así vamos a poder salir adelante en esta época tan cambiante, tan dinámica. Generar una sociedad menos tensa, menos estresada, una sociedad conformada por personas que trabajen por la propia persona, por eso hablo de una pedagogía de la persona, una alternativa de futuro es una pedagogía de la persona que lleve a autorregularse, a automotivarse, auto educarse y ser más responsables consigo mismo, porque no va a poner en otros sus metas, sino en su propio compromiso y responsabilidad total.

Gracias por la entrevista; puedo, finalmente, reafirmar que una Universidad de la Mujer nos permite cumplir con nuestra misión de formar mujeres profesionales, capaces de transformar nuestro querido país, ser agentes de cambio, sin perder nuestra condición de personas responsables, éticas, que este es nuestro principal compromiso con la sociedad. 